From the author:

G.F. Sterrazza Papa and co-workers raise valid concerns regarding excessive radiation exposure from chest computed tomography (CCT), which is an area of growing interest for both the medical community and the general public. It is correctly pointed out that multiple scans may mean a significantly increased lifetime attributable risk of cancer when estimated from prior studies of atomic bomb survivors and workers in the nuclear industry [1]. However, it would be remiss to not point out that the risk of cancer from a CCT is not particularly daunting in adults aged $>50$ yrs with $<0.02 \%$ lifetime attributable risk per abdominal $\mathrm{CT}$; this is comparable in radiation exposure to our institutions CT pulmonary angiography and less than our non-contrast CCT. As the PORT study found that $74.6 \%$ of 1,343 in-patients were aged $>50 \mathrm{yrs}$, it seems likely that CCT would be a fairly safe modality in the majority of hospitalised pneumonia patients [2]. It must be kept in mind that radiation exposure is primarily of concern in younger patients, particularly those aged $<20 \mathrm{yrs}$, where ultrasound is already recommended by the British Thoracic Society [3].

Regardless of the probably negligible cancer risk attributable to radiation exposure in the majority of elderly pneumonia patients, the indiscriminant use of CCT in the assessment of parapneumonic pleural effusion would neither be medically wise nor a judicious use of medical resources. Other techniques such as ultrasound provide a radiation free and readily accessible alternative. It was not our intention to suggest that CCT should replace other means of assessing a pleural effusion. However, whether for good or bad the use of CCT in the USA has increased from 20 million scans per year in 1995 to 62 million by 2005 [1]. As our paper demonstrated, $40 \%$ of our admitted pneumonia patients received CCT (mostly CT pulmonary angiography), not for pleural effusion assessment but for the initial evaluation of hypoxia and exclusion of pulmonary embolism while in the emergency room [4]. We think it is important to clarify that the intent of our article was to provide a way to assess the need for thoracentesis when a CCT was already obtained in order to expedite patient care and reduce the cost and radiation exposure of additional testing, and is not suggesting that CCT should become the first line means of assessing parapneumonic pleural effusions.

\section{B.K. Moffett}

Correspondence: B.K. Moffett, Dept of Internal Medicine, University of Louisville School of Medicine, 800 Zorn Avenue, Louisville, 40206, KY, USA. E-mail: bryan.moffett@va.gov

Statement of Interest: None declared.

\section{REFERENCES}

1 Brenner DJ, Hall EJ. Computed tomography - an increasing source of radiation exposure. $N$ Engl J Med 2007; 357: 2277-2284.

2 Fine MJ, Auble TE, Yealy DM, et al. A prediction rule to identify low-risk patients with community-acquired pneumonia. $N$ Engl J Med 1997; 336: 243-250.

3 Balfour-Lynn IM, Abrahamson E, Cohen G, et al. BTS guidelines for the management of pleural infection in children. Thorax 2005; 60: Suppl. 1, i1-i21.

4 Moffett BK, Panchabhai TS, Anaya E, et al. Computed tomography measurements of parapneumonic effusion indicative of thoracentesis. Eur Respir J 2011; 38: 1406-1411.

\title{
What the pulmonary specialist should know about the new inhalation therapies
}

\section{To the Editors:}

In the recent report of the European Respiratory Society/ International Society for Aerosols in Medicine Task Force [1], the authors correctly identify the need to prescribe spacers (sometimes termed valved holding chambers (VHCs) when an inhalation valve is present) to medication delivered from pressurised metered-dose inhalers (pMDIs). Although they mention that some pMDI products are licensed for use with a particular spacer, they have failed to point out that each pMDIspacer combination should be treated as a unique system.

In 2008, the UK Medicines and Healthcare Products Regulatory Agency (MHRA) published a recommendation that for inhaled corticosteroids (ICSs), "Spacers should not be regarded as interchangeable: patients who use a spacer with their inhaler should use the spacer device named in the Summary of Product Characteristics" [2]. This guidance further stipulates
"Patients whose asthma is well-controlled and who are using a spacer should always use the same type of spacer and not switch between spacers. Different spacers may deliver different amounts of inhaled corticosteroid, which may have implications for both safety and efficacy" [2].

The following year, the European Medicines Agency (EMA) set out the requirements concerning clinical documentation for orally inhaled products (OIPs) for use in the treatment of asthma and chronic obstructive pulmonary disease [3]. This European-wide guidance specifies, "When all data collected in the development programme are based on the product administered via a pMDI together with one or more specific, characterised spacers, the product can be authorised subsequently for use only if used with the specific named spacer(s)." Shortly after publication of this guidance, DISSANAYAKE [4] (formerly a Medical Assessor at the MHRA) provided an interpretation of the EMA guidance as follows: " ....given that a 